\title{
Las mujeres como objetos transccionales en la instauración de sociedades androcráticas
}

\author{
Women as transctional objects \\ in the establishment of androcratic societies
}

Estela Andrea Serret Bravo

Recibido: 16/03/2018

Aceptado: 22/04/2018

\section{RESUMEN}

El artículo intenta demostrar, a través de un análisis teórico y de la etnografía comparada, que la definición de las mujeres y los hombres en cada contexto socio histórico, depende radicalmente de las definiciones culturales que ambos grupos obtienen en el complejo entramado de intercambios simbólicos, o pactos patriarcales, que da cuerpo a una comunidad en específico, y a sus relaciones primarias de dominación. En este sentido, una cierta caracterización de las mujeres, entendidas siempre como objetos transaccionales, es indispensable para la definición específica del varón prototípico que produce cada sociedad.

Palabras clave: género; pactos patriarcales; identidades de género; androcracia; objectos trnasaccionales

\begin{abstract}
The article tries to demonstrate, through a theoretical analysis and comparative ethnography, that the definition of women and men in each socio-historical context depends radically on the cultural definitions that both groups obtain in the complex network of symbolic exchanges, or patriarchal pacts, which gives body to a specific community, and its primary relations of domination. In this sense, a certain characterization of women, always understood as transactional objects, is indispensable for the specific definition of the prototypical male that each society produces.
\end{abstract}

Keywords: gender; patriarchal pacts; gender identities; androcracy; transactional objects

Estela Andrea Serret Bravo es doctora en Filosofía Política por la Universidad Nacional de Educación a Distancia (UNED, Madrid, España). Profesora investigadora titular del Departamento de Sociología de la Universidad Autónoma Metropolitana Azcapotzalco (México). Correo electrónico: estelaserret@hotmail.com. ID: https:/ / orcid.org/0000-0001-9878-2677

Cómo citar este artículo: Gimeno, B. (2018). Las mujeres como objetos transaccionales en la instauración de sociedades androcráticas. Atlánticas. Revista Internacional de Estudios Feministas, 3 (1), 175-191 doi: http:/ / dx.doi.org/10.17979/arief.2018.3.1.3394 


\section{ESTADO DEL ARTE}

Para el pensamiento feminista la pregunta ¿qué es una mujer? ha sido clave desde sus orígenes. Las respuestas esencialistas, humanistas o de corte semejante, no me ocuparán en este texto. En cambio, al plantear ese cuestionamiento desde un enfoque distinto creo que podemos encontrar algunas claves para descifrar el papel que juega la definición de las mujeres en la construcción de los órdenes androcráticos.

En un texto de sobra conocido, Gayle Rubin plantea en 1975 (Cf. Rubin, 1975) el tema desde la perspectiva que ahora me interesa. Al examinar en qué sentido puede serle útil el enfoque de la antropología a las preguntas feministas, Rubin comprende que el estudio estructural de las relaciones de parentesco en las sociedades tribales proporciona claves inestimables para comprender que cada sociedad construye significados específicos de lo que hace a un verdadero hombre y a una verdadera mujer; que esos significados varían enormemente entre una sociedad y otra (y en momentos históricos diferenciados).

Si revisamos por nuestra cuenta los datos etnográficos veremos, por ejemplo, que la sociedad arapesh considera muy masculino que los padres padezcan el embarazo y el parto junto con sus esposas y que participen activamente en la crianza temprana de su descendencia tan activamente como ellas. Ese mismo pueblo valora poderosamente la dulzura y sutileza en sus varones, quienes tienen conductas y actitudes que la estadounidense Margaret Mead consideraba en la década de 1930 marcadamente femeninas (Mead, 1982). Junto con esto, la propia observación antropológica de la primera mitad del siglo XX daba cuenta de cómo muchas personas, fisiológicamente femeninas, ocupaban entre los nuer, del África central, el papel de hombres, con los correspondientes roles de dueños de ganado, guerreros, esposos y padres de las criaturas que sus esposas engendraban con ayuda de amantes útiles. La relación con el ganado de estas hembras biológicas que encarnan hombres sociales, es típicamente masculina, y eso, entre los nuer, tiene una importancia simbólica decisiva:

"Cattle have historically been of the highest symbolic, religious and economic value among the Nuer: the difference between people and cattle is continually underplayed. Cattle are particularly important in their role as bride wealth, where they are given by a husband's lineage 
to his wife's lineage. It is this exchange of cattle which ensures that the children will be considered to belong to the husband's lineage and to his line of descent. The classical Nuer institution of ghost marriage, in which a man can "father" children after his death, is based on this ability of cattle exchanges to define relations of kinship and descent. In their turn, cattle given over to the wife's patrilineage enable the male children of that patrilineage to marry, and thereby ensure the continuity of her patrilineage. A barren woman can even take a wife of her own, whose children (obviously biologically fathered by men from outside unions) then become members of her patrilineage, and she is legally and culturally their father, allowing her to participate in reproduction in a metaphorical sense" (Hutchison, 1992: 310).

O, por citar un último ejemplo que sorprendía (y, muy probablemente, escandalizaba a los antropólogos que lo narraron) el caso del pueblo azande en el norte del Congo. La estructura social azande contemplaba como legítimo que, en ciertos contextos especiales, los hombres jóvenes tomaran a otros hombres jóvenes, hasta ese momento considerados perfectamente masculinos por su comunidad, como esposas. A partir de la ceremonia de matrimonio todo su entorno se refería a estas nuevas mujeres con términos e ideas específicamente empleados para designar a las féminas de toda la vida y su sitio en la estructura social, así como su atuendo, sus conductas y sus expectativas se tornaban enteramente femeninas. (Evans-Pritchard, 1937). También Ruth Benedict alude a este tipo de inversiones -según los patrones de la cultura occidentalpresentes entre los pueblos nativos de Norte América:

"Entre muchas tribus indias norteamericanas existe la institución del berdache (12, 15), como los franceses la han llamado. Estos varonesmujeres eran varones que en la pubertad o más tarde adoptaban la vestimenta y las tareas femeninas. En ocasiones se casaban con otros varones y vivian con ellos. A veces se trataba de varones sin inversión sexual, personas con dotaciones sexuales débiles que elegían este rol para evitar las burlas de las mujeres" (Benedict,1932:5).

Estos análisis comparativos permiten a Rubin y muchas otras antropólogas feministas de su época, insistir en dos puntos sustantivos ${ }^{1}$. Primero, que, pese a

\footnotetext{
${ }^{1}$ Recupero estos dos fuera de la concatenación deductiva en que los inscribe su autora, porque hay algunos supuestos en esa lista que los argumentos de este artículo no pueden compartir.
} 
la intuición compartida en contrario, los caracteres, gustos, actitudes, gestualidad, habilidades, sueños, ideales y papeles sociales que pensamos distinguen a las personas en hombres y mujeres, por naturaleza, son en realidad definidos y ritualizados simbólico/imaginariamente, de manera distinta en cada contexto particular.

Esta primera idea se refuerza cuando observamos otro marcador de género claramente distinguible en las sociedades tribales: la división sexual del trabajo. En efecto, también ya había sido demostrado por Lévi-Strauss (1969:291-313) que, si bien la prohibición de que los hombres realicen tareas de mujeres y viceversa es estructural, la forma en que se manifiesta varía ostensiblemente de un contexto socio cultural a otro. Como recuerda Sherry Ortner, si en una sociedad las mujeres pescan y los hombres se dedican a la agricultura, en la comunidad vecina podemos constatar exactamente lo contrario. Y, con este ejemplo, atendemos al segundo punto crucial: no importa cómo sean definidos los hombres y las mujeres ni cuáles se piense que son sus actividades características, en todos los casos se considerará que esos rasgos y esas actividades les corresponden por naturaleza. $\mathrm{Y}$ siempre los atributos considerados masculinos gozarán de mayor prestigio (y proporcionarán mayor poder) que los femeninos (Ortner, 1979).

Ahora bien, si regresamos a nuestro punto de partida, la pregunta subsiste; más allá de las particularidades que en cada caso la definan, ¿qué es una mujer? $\mathrm{O}$, de otro modo, como he intentado precisar desde hace unos años, ¿qué es una persona cuya asociación con lo femenino, sin importar nada más, es socioculturalmente desvalorizada?

En el texto de referencia la propia Rubin nos da algunas pistas, siguiendo, de nuevo, a Lévi-Strauss. Este autor, inspirado, a su vez, en Marcel Mauss (Mauss,1979), comprende que las mujeres juegan un papel fundamental en la organización misma de las relaciones sociales del mundo tribal (y no solo de él) por cuanto constituyen los bienes a través de cuyo intercambio los hombres de una comunidad construyen vínculos de distinto tipo. La relación más característica que se construye a través del intercambio de mujeres es la de matrimonio. Como puede apreciarse por esta formulación, el matrimonio se descubre, no como una institución que vincula a un hombre (o varios) y una mujer (o varias), sino a dos varones. Son efectivamente ellos quienes, al dar y 
recibir mujeres, edifican familias, linaje, estructuras económicas, alianzas, acuerdos políticos; pagan tributos; resuelven conflictos bélicos, $\mathrm{y}$, en definitiva, construyen relaciones sociales de todo tipo. En su descripción acerca de las formas tribales de familia, Lévi-Strauss constata una valoración desigual de los hombres y las mujeres que se evidencia, por ejemplo, en las relaciones polígamas y en los derechos de autoridad que, aún en las familias matrilineales, son ejercidos por hombres (en estos últimos casos, por los hermanos de la madre). En el análisis de las relaciones familiares de estos pueblos se habla de las mujeres claramente como si se tratara de pertenencias de los hombres:

"(...) si consideramos el caso de algunas tribus australianas (...) podemos darnos cuenta de que un hombre que se mostrara reacio a prestar a su esposa durante las ceremonias religiosas sería considerado <muy egoísta> ya que trataría de monopolizar un privilegio que el grupo social considera que debe compartir..." (Lévi-Strauss, 1987:12).

Cuando se habla de poliandria, por otro lado, no se piensa nunca que una mujer tenga acceso a varios maridos, sino que varios hombres comparten una esposa (cf. ibid, :14). Nuestro autor da por hecho que la división sexual del trabajo es condición de existencia de las sociedades humanas, pero no explica por qué en todas estas sociedades, cualquiera que sea la forma concreta con que tal sanción existe, se desvaloriza siempre el trabajo realizado por las mujeres. Él mismo admite como dudosa la idea, vigente en su época, de que haya actividades que se asignen universalmente a uno $\mathrm{u}$ otro sexo, como la cacería a los hombres y el parto y la crianza a las mujeres (no podemos menos que asombrarnos de que nuestro estructuralista considere un trabajo social y cultural el dar a luz, y debemos señalar que quizá lo hace porque ha atestiguado cómo, en algunas sociedades, hay hombres obligados a simular el trabajo de parto; cf. LéviStrauss, 1987: 31). Hoy sabemos que la cacería también ha sido practicada por mujeres y que hay pueblos donde la crianza es tarea masculina -compartida con las mujeres o exclusiva de los hombres- (cf. Martínez Pulido, 2006:140-167). A diferencia de la falta total de sorpresa que nuestro autor demostrara frente a estos hechos al dibujar su organización fundamental en Las estructuras elementales del parentesco, en el artículo que ahora analizamos (La familia) acusa recibo de lo bizarro que, para un pensamiento racionalista congruente, puede resultar la cosificación de la mitad del género humano. Así, nos brinda la siguiente reflexión: 
"La lectora que se siente horrorizada al ver que las mujeres son tratadas como mercancía sometida a las transacciones controladas por grupos de hombres, puede consolarse fácilmente con la seguridad de que las reglas del juego no cambiarían si consideráramos grupos de mujeres que intercambian hombres" (Lévi-Strauss, 1987:46).

Pero lo cierto es que esta última posibilidad no sólo es una quimera por su inexistencia histórica sino que, como hemos procurado demostrar, lo que hace que un grupo de personas sea pensado como mujeres es, justamente, la parcial deshumanización de la que son objeto al encarnar significados de feminidad. Y es precisamente eso lo que permite que los varones (grupo definido como el propiamente humano, en el que su anatomía sexual es solamente un subrogado de masculinidad, que puede eventualmente, ser cambiado por otro elemento simbólico) sean necesariamente los sujetos que intercambian y las mujeres los objetos de esa relación. En otras palabras: no puede imaginarse un grupo social en el que la regla sea que las mujeres intercambien hombres, porque parte de lo que hace a un hombre es su capacidad de intercambiar mujeres, y viceversa; un elemento fundamental en la definición de alguien como mujer es que encarna un objeto transaccional, a través de cuyo intercambio, se definen las relaciones sociales.

Estos hechos, que Lévi-Strauss constata sin asombro y Rubin problematiza, nos arrojan pues, una primera respuesta a nuestra pregunta inicial: al menos en las sociedades tribales, las mujeres son bienes valiosos cargados de poder simbólico y que no pueden ser reducidos a otro tipo de bienes, que los hombres intercambian para construir relaciones sociales. Esta idea nos pone sobre la pista de una certeza sobre la que ya alertara Simone De Beauvoir: la mirada cultural considera solo a los varones plenamente humanos; ser mujer implica encarnar una identidad parcialmente deshumanizada.

En otro texto feminista ya clásico, y desde una preocupación diferente, Carole Pateman (Pateman, 1992) regresa a tematizar los vínculos entre varones establecidos a través del intercambio de mujeres, con otra terminología y enfocándose en un tipo distinto de sociedad. Se trata, como puede adivinarse, de su propuesta sobre la historia no contada del contrato social hipotético con el que la filosofía política explica los fundamentos normativos de las sociedades 
modernas. Como recordaremos, Pateman demuestra que solo la previa (en términos lógicos) realización de un contrato sexual entre varones para acordar las normas de acceso sexual a las mujeres puede explicar los términos de aquel famoso pacto. En esta perspectiva, las mujeres no se perciben como bienes que son poseídos por los varones en virtud de su nacimiento en el seno de una familia sino como un hipotético genérico descontextualizado que ha de ser sometido por otro genérico de manera integral. Para Pateman, el supuesto no dicho, pero indispensable, que antecede al contrato social, signado, como se sabe, por individuos libres e iguales, es el acuerdo entre todos los varones del mundo burgués para actuar como una fratría al equiparar el derecho de todos al disfrute sexual de las mujeres. Con este acuerdo, los hermanos simbólicos del mundo burgués, habrían cambiado las reglas del patriarcado tradicional, en el cual el padre/jefe monopolizaba para sí el derecho sobre las mujeres. Tal situación de igualdad es la que permite a los hermanos simbólicos anular el principio del estatus y re-conocerse como iguales. La propia categoría de individuo, sería así, según nuestra autora, necesariamente masculina, y sólo podría calificar a quienes su cuerpo sexualmente diferenciado hace hombres. Una vez constituidos en individuos gracias al contrato sexual, los hombres crean el mundo civil en el cual la forma reglamentada de acceso a las mujeres es doble: de manera serializada por medio del casamiento, o colectiva a través de la prostitución. En ambos casos, el mecanismo es un curioso contrato que requiere, sí, el consentimiento de ambas partes (un hombre y una mujer), pero en el cual los contratantes ya son, incluso antes de pactar el intercambio de obediencia por protección (en el matrimonio) o de acceso sexual por una paga (en la prostitución), desiguales entre sí. En términos de Pateman, ellos dominadores, ellas dominadas. La razón de esta paradoja aparente se halla en que el pacto no dicho pero que precede y condiciona la prostitución $\mathrm{y}$ el matrimonio es el que los varones signaron previamente entre sí. Es decir, la lógica de estos acuerdos imaginarios indica que antes de la creación de la comunidad política burguesa a través del pacto entre individuos/varones, estos firmaron un pacto específico para, desde el mismo Estado de Naturaleza, acordar las reglas de apropiación sexual de las mujeres. Este contrato sexual no puede hacerse explícito, porque, de hacerlo, los divulgadores del ideal iusnaturalista se verían obligados a reconocer que el sometimiento de todas las mujeres a todos los varones se legitima en virtud de que consideran que ellos tienen tal derecho por naturaleza. Hacer visible este supuesto es impensable, pues ellos son los amos en un mundo fundado sobre la idea de que las 
jerarquías no se establecen por desigualdad natural sino por acuerdos racionales. Por ello también, debe decirse, se instaura en el imaginario la ficción de que la subordinación de las mujeres sólo se concreta en la medida que signan, una a una, el contrato de matrimonio o el de prostitución, cuando en realidad, como cualquier soltera no prostituta ha experimentado, la subordinación del conjunto de las mujeres al conjunto de los varones subsiste en el imaginario social moderno (y es justamente ésta la que tematiza Pateman con la idea del contrato sexual).

Vemos que el vínculo más importante que estos contratos sellan es el de los hombres reconvertidos en fratría a través del procedimiento, explicado brillantemente por Celia Amorós (Amorós, 2009), de homologar a las mujeres y hacer a cualquiera de ellas susceptible de apropiación por cualquier hombre, con independencia de su estatus. Pero, ¿qué imaginada condición ontológica permite al genérico masculino dominar al conjunto de las mujeres? Lamentablemente, la respuesta de la filósofa británica nos regresa a la presunta objetividad del cuerpo sexuado. La seriedad con la que Pateman considera que las capacidades reproductivas diferenciadas hacen a los hombres, hombres y mujeres a las mujeres, se revela en su reflexión sobre la maternidad subrogada.

Así, su óptica sobre el tema de los vínculos establecidos entre varones a través de su acceso a las mujeres es distinta de la que interesaba a Gayle Rubin no solo porque se trata de una mirada filosófico política, normativa, versus otra antropológica, explicativa, sino especialmente porque aquélla reifica los géneros anclándolos a una idea mistificada de la materialidad del sexo. Con todo lo que la brillante contribución de la autora del Contrato Sexual brinda a la teorización feminista sobre, por ejemplo, las fallas estructurales en la conformación de las democracias modernas, su recuento de los mecanismos que permiten la instauración de las mujeres como objetos de apropiación masculina en el contrato sexual no nos acerca lo suficiente a la resolución de nuestro problema, pues, ya lo habían mostrado Rubin y muchas más: una mujer no es aquella que puede parir si hallamos tantas que son mujeres y esposas sin úteros ni vaginas y tantos hombres capacitados para embarazarse por la tan cacareada naturaleza. No obstante, la productividad teórica y analítica de las enseñanzas de Pateman no se frena por esta derivación. Así lo prueba el que Celia Amorós recurra a lo que aquélla nos enseña sobre el papel de los dos contratos originarios, el sexual y el social, en la conformación de los imaginarios de la modernidad, para 
ofrecernos una reflexión más general sobre las formas específicas en que distintos pactos patriarcales engendran distintos órdenes sociales. Su perspectiva nos permite regresar a pensar teóricamente qué es una mujer (y, por lo demás, qué es un hombre) desde una mirada interpretativa que no encuentre su freno en el muro de ese fetiche que es el cuerpo sexuado.

También la antropología, en su intersección con el psicoanálisis, nos permite comprender cómo una mirada materialista puede, al mismo tiempo, sostener que el cuerpo (en su dimensión sexual y en todas las demás) es tallado, modelado por las culturas particulares. En efecto, para poner sólo un ejemplo de esta posibilidad, hay que atender de nuevo a Lévi-Strauss, quien al respecto nos dice:

"Las capacidades de exitabilidad, los límites de la resistencia son diferentes en cada cultura; los esfuerzos <irrealizables>, los dolores <insufribles> (...) están menos en función de las particularidades individuales que de los criterios sancionados por la aprobación o desaprobación colectiva. Cada técnica, cada conducta aprendida y transmitida por tradición, están en función de ciertas sinergias nerviosas y musculares que constituyen cada una un verdadero sistema, solidario por otra parte, con un determinado contexto sociológico" (Lévi-Strauss, 1979:15).

Amorós dirige su reflexión de nuevo al problema (para el feminismo) del intercambio de mujeres, pero, al ser precisado por el concepto de que tales intercambios se realizan siempre según la lógica de los pactos patriarcales, nuestra autora explica, con toda justeza, que las mujeres juegan en ellos el papel de objetos transaccionales. Esa sería, de hecho una definición general y adecuada, y una primera aproximación a nuestra buscada respuesta: una mujer es una persona (en lo real) que (no obstante) es interpretada (y auto interpretada) como un objeto transaccional a través de cuyo intercambio se producen vínculos entre (quienes son entendidos como) varones. Ahora, lo que nos permite la propuesta de Amorós es hacer de esa respuesta general un razonamiento progresivamente específico en tanto que ella misma da cuenta de cómo los términos de tales pactos varían de acuerdo con los distintos contextos histórico culturales, involucrando, en cada caso, el tejido de complejas redes significantes de género. Recupero aquí dos de las ideas con las que nuestra autora nos abre la puerta a la reflexión que me interesa: Primero, que las 
mujeres funcionan como el objeto transaccional de los pactos que tejen los varones y que revisten modalidades muy diferentes, dependiendo del contexto en que se produzcan. Segundo, derivado de lo anterior, que existen relevos histórico situacionales en las designaciones que los varones hacen de ellos mismos y, consecuentemente, en las designaciones de hacen de las mujeres (Cf. Amorós, 2008: 218 y 219).

De acuerdo con estas coordenadas generales, la autora explica la forma que adoptan dos modelos de pactos patriarcales que se oponen a la sociedad del contrato. Para clarificar cómo y en qué se oponen a la sociedad burguesa los que Amorós llama imaginario libertino e imaginario mafioso, comenzaremos por recordar los principios androcráticos que instituyen aquélla.

Señalemos de entrada que el individuo, modelo masculino prototípico de la sociedad moderna, no logra imponerse como representación hegemónica de masculinidad sin lucha. Precisa defender esa opción en contra de la figura masculina paradigmática de las sociedades estamentales. En oposición a esa figura, marcada por la estratificación natural, la sociedad burguesa en consolidación debe imponer el concepto del hombre nuevo: aquél que nace tan libre como su semejante y a quien la naturaleza autoriza a subordinarse sólo si lo hace a una ley racional. Esto nos muestra, desde luego, que la definición de lo que hace a un hombre ser tal varía de una sociedad a otra, y que esas definiciones diferentes se toman como naturalmente verdaderas en cada contexto. Las representaciones sociales del varón paradigmático aparecen y se reproducen en todo tipo de discurso social; mítico, religioso, experto, filosófico, científico, estético, esotérico, pedagógico, cotidiano, etcétera.

Así, para que existan individuos libres e iguales, los varones modernos tienen que constituirse en tales. ¿Cuál es el proceso (hipotético) mediante el cual los varones dejan de ser los de la sociedad estamental o despótica y pasan a conformarse como individuos? ¿Qué tiene que ocurrir para que se dé este paso? Lo que nos diría Amorós es que la condición necesaria para que los varones dejen de estar diferenciados por estamentos y puedan empezar a ser individuos libres e iguales, es que firmen un pacto en el que cambien las reglas de apropiación de las mujeres (Amorós,2009). Ese es precisamente al que llama Pateman el contrato sexual. El que da pie a la conformación de individuos (es decir, la forma imaginaria en que se modela la masculinidad en la sociedad 
moderna) es un pacto en el que los varones, convertidos en hermanos simbólicos, pactan tornar a las mujeres en idénticas. Es decir, pactan cancelar la estratificación de las mujeres quienes, en la sociedad estamental, se dividen, por ejemplo, en princesas y plebeyas. Mediante el acuerdo, los varones redefinen a las mujeres, en primer lugar, al conformarlas a todas como bienes idénticos, susceptibles de apropiación por cualquier varón miembro de la fratría. Lo que hace a los hombres iguales, pues, es la desaparición de las condiciones desiguales de apropiación de las mujeres. Porque la sociedad pre moderna, estamental, mantiene entre las distintas mujeres categorías muy específicas que las marcan como sólo susceptibles de apropiación por determinados varones. Las mujeres en sociedades estamentales no son propiedad pro indiviso de todos los varones, sino que pertenecen a una élite de hombres o incluso a un sólo hombre. De modo que para establecer lazos de lealtad, de honor, de vasallaje, los poderes específicos distribuyen a las mujeres en usufructo, ni siquiera en propiedad; ésta solamente la detenta el señor feudal, el rey, $u$ otra figura semejante. La tesis de Amorós nos muestra que en las sociedades modernas las mujeres dejan de tener categoría (como ya indicara Napoleón, según nos recuerda nuestra autora); sólo adquirirán una en virtud del varón que las posea. Las verdaderas mujeres en la sociedad burguesa, se dividen en dos tipos: uno, visible, normativo, el de la mujer doméstica: esposa, madre y ama de casa que, según nos dice Rousseau, permitirá al varón que se ha apropiado de ella mediante el contrato de matrimonio existir como individuo y ciudadano. Las mujeres solteras, según este modelo, se preparan en su infancia y juventud para ser elegidas por un varón y llegar a ejercer como ángeles del hogar. El segundo tipo, invisible para el discurso normativo pero clave para la consolidación de los pactos patriarcales, es la prostituta. Mujer pública, será sujeto de uso temporal (no se le adquiere en propiedad sino se le alquila) por cualquier varón que esté dispuesto a signar el convenio comercial requerido. Frecuentemente ese otro contrato se efectúa también entre dos varones: en este caso el proxeneta y el cliente. Como en el caso anterior, el del contrato de matrimonio, se requiere formalmente la aquiescencia de la mujer objeto del pacto, lo cual vuelve a confirmar que, aun cosificadas, las mujeres nunca pueden ser pura naturaleza, puro objeto o pura animalidad. Su carácter especialmente valioso deriva de su representación imaginaria como seres que encarnan los límites entre naturaleza y cultura; de su ambigüedad simbólica; de su densidad significante.

Volviendo al acto fundacional del contrato sexual burgués, es justamente la 
definición precisa de las mujeres como idénticas y sin categoría, objetos de apropiación serializada o colectiva, lo que posibilita la definición de los varones modernos como individuos iguales (Cf. Amorós, 2009).

Ahora bien, este mecanismo de erección de la masculinidad modélica no es privativo de la sociedad burguesa. Los proyectos antagónicos del orden moderno, como el libertino o el mafioso tematizados por Amorós, requieren de la respectiva redefinición de las mujeres. Cuando un grupo emergente somete a crítica la idea de mujeres que la sociedad anterior ha sostenido, y establece nuevas reglas para su intercambio y apropiación, también está revelando cuál es su noción de hombre verdadero.

En su descripción del imaginario libertino, construido por el Marqués de Sade, Amorós reconstruye la lógica compleja y paradójica de este modelo patriarcal antiburgués. Sade resulta un contradictorio hijo de la Ilustración y el Antiguo Régimen, que se rebela contra ambos a través de la transgresión. Para ello, prescribe el rechazo categórico a la feminidad normativa del orden ilustrado: la mujer doméstica. La madre abnegada, la mujer virtuosa de pureza angelical, se convierte en el objeto privilegiado de la transgresión libertina. No sólo exige Sade que tal "Mujer" sea desacralizada, sino virtualmente destruida. Contra la regla burguesa de apropiación serializada de las mujeres por medio del contrato de matrimonio, el libertino, confrontado con la aristocracia tanto como con la burguesía, no establece orden alguno, sino que pregona la continua transgresión:

[...] la perpetua transgresión -que no sustitución- de sus reglas [las del Padre] requiere un ritual propiciatorio asimismo permanente y que no va a consistir sino en el sacrificio de una víctima inocente. [Sade pone a las mujeres] en medio y en común, a disposición de un genérico masculino tiránico no constituido en fratría y que, por tanto, no ha dispuesto reglas de acceso a las mismas: "todas para todos" (Amorós, 2008: 230).

Sade niega la libertad burguesa y la desplaza hacia el deseo, y en esta paradoja (pues el deseo implica amarras ${ }^{2}$ ) la hace depender de la anulación del objeto de

\footnotetext{
${ }^{2}$ El deseo, efectivamente, ata, no libera. Una verdad básica que, como apunta brillantemente Ana De Miguel (comunicación personal), niega ferozmente la modernidad. Esta era fetichiza el deseo y crea la ilusión de que en él hallamos la libertad.
} 
ese deseo. Las mujeres, definidas como objeto de deseo, deben ser metódicamente destruidas (a través de la violación tumultuaria, la sodomización, la repartición incesante entre todos los varones, la inmolación y el sacrificio) para liberar al deseo de las ataduras a su objeto. El deseo libertino se vuelca entonces sobre sí mismo instituyéndose como deseo del deseo. Así, el orden burgués se cambia por el desorden transgresor fracturado entre el linaje y la igualdad.

En un contexto muy diferente, el de la sociedad juarence en el norte del México contemporáneo, vemos aparecer otro modelo de pacto patriarcal antiburgués en el que Amorós descubre tintes del imaginario libertino. Dadas sus características específicas, nuestra autora decide bautizarlo como imaginario mafioso. Éste surge en una situación social en la que, si bien formalmente se reconoce regida por las reglas del contrato burgués, sus instituciones, dramáticamente frágiles, se encuentran gobernadas por poderes corruptos. Son estos últimos los que dan cabida al fortalecimiento de las mafias que acaban por funcionar como verdaderos estados paralelos. En la medida que su poder se afirma, debe ser refrendado por categorías de interpretación del mundo que pujan por triunfar en el imaginario social. Amorós nos indica algunas de las claves del imaginario mafioso:

La mafia representa una voluntad de reinstitución del mundo del status, de los privilegios despóticos, en el mundo del contrato y de los derechos. Conlleva la imposición de una familia simbólica que se infiltra e incrusta en el espacio público del contrato social y lo fagocita. $Y$ ha de hacer lo mismo, por otra parte, con el ámbito privado familiar de las relaciones de sangre. No es de extrañar, en estas condiciones, que sus vínculos juramentados consistan en un dar, tomar y sellar la palabra con sangre que es a la vez literal y simbólica. [...] el Padre es sustituido por el Padrino y se puede llegar a exigir al candidato que quiere ingresar en la red de los pactos mafiosos la inmolación de un miembro -hermana, madre- de la familia natural, en el ritual iniciático de adopción. La sangre sin linaje [...] sella metonímicamente los vínculos mafiosos. Y como las mujeres somos el objeto transaccional de los pactos entre varones, la sangre femenina es la candidata por excelencia para sellarlos (Amorós, 2008:244). 
Los pactos mafiosos requieren generar una definición de mujer -opuesta a la del imaginario burgués- que se hace evidente cuando vemos los cuerpos secuestrados, violados, torturados, desmembrados y expuestos al ojo público. Así, aquello que las mujeres y los hombres son para cada contexto socio histórico, depende radicalmente de las definiciones culturales que ambos grupos obtienen en el complejo entramado de intercambios simbólicos que da cuerpo a una comunidad en específico. $\mathrm{Y}$ a sus relaciones primarias de dominación.

Recapitulando: todo orden social se funda en consensos implícitos: códigos simbólico-imaginarios que ordenan los sistemas de mando de acuerdo con una idea del mundo. Esos consensos funcionan cotidianamente como la ritualización de pactos entre varones. A través de esos pactos imaginarios los hombres de cada cultura se definen a sí mismos y a su sociedad. Las sociedades con un sistema de dominación masculina (androcráticas o patriarcales) no conciben a las mujeres como plenamente humanas, sino como bienes valiosos gracias a cuyo intercambio los hombres se convierten en tales. En ese sentido, las mujeres son, en primer lugar, objetos transaccionales que permiten los intercambios fundacionales entre varones. Cada sociedad tiene sus propias reglas, sus propias estructuras de mando y, en consecuencia, su propia definición de cómo son los "verdaderos hombres". Esa definición se logra sólo si previamente (en términos lógicos) se acuerda la definición y el valor que se asignará a los objetos de esos pactos: las mujeres. En las coyunturas de cambio social, cuando un grupo lucha por establecer su dominación desplazando a otro(s) de los centros de poder, resulta clave que el grupo emergente se manifieste descalificando la definición aceptada de "mujer" y posicionando su propia designación. Estos imaginarios en competencia son decisivos para fincar la hegemonía de un nuevo grupo sobre la del anterior. Se ejemplifica cómo se produce esa competencia semántico-política a través del análisis que Celia Amorós emprende para explicar la lucha entre distintas visiones del mundo, expresadas como otros tantos pactos patriarcales (según la propuesta de Carol Pateman): la oposición entre la sociedad del contrato y la del estatus; la descalificación de aquella por el ideario libertino, primero, y por la sociedad mafiosa después.

Las teorías del contrato sexual y de los pactos patriarcales que tan brillantemente proponen Pateman y Amorós nos permiten iluminar, como se 
ve, el asunto complejo que nos ocupa; pensar en las consecuencias que tiene la definición simbólico imaginaria que las distintas sociedades hacen de mujeres y hombres. Si bien esa definición concreta presenta variaciones considerables según qué sociedad y qué tiempo histórico se revise, existe un punto de partida, empíricamente constatable, que todas comparten. Se trata de la concepción que agrupa a las personas en dos grandes categorías: los seres humanos y los bienes transaccionales o monedas simbólicas (no plenamente humanas, no plenamente animales) a través de cuyo intercambio los hombres se construyen como tales. Los intercambios de mujeres toman la forma de acuerdos simbólicos entre hombres -categoría que, como sabemos, se identifica transculturalmente con el genérico humano-. Las mujeres, por su parte, al funcionar en los distintos pactos como objetos transaccionales sólo se realizan como tales objetos en el acto performativo del intercambio. Su función es conformar a quienes realizan el intercambio como sujetos. Un ejemplo característico lo encontramos en un aforismo del pueblo arapesh que recupera Margaret Mead:

"Tu propia madre/ tu propia hermana/ tus propios puercos/ tus propios ñames que tú has apilado/ no puedes comerlos. Las madres de los demás / las hermanas de los demás / los puercos de los demás / los ñames que los demás apilaron/ puedes comerlos" (Mead, 1982:81).

La retórica elegida para formular este mandato arapesh nos muestra con claridad una lógica que subyace a todas las categorizaciones patriarcales de las mujeres: ellas son bienes preciosos, que se distinguen de otros bienes en que su propia definición y las normas de su intercambio diseñan la estructura específica de un orden determinado. Desde este punto de vista, mujeres son aquellas a través de cuyo comercio se definen los seres humanos, esto es los hombres, quienes encuentran así su definición, sus límites y su concreción precisa. Pero, a la vez, son aquellos bienes que, cuando ajenos, conviene destruir y degradar para, así, atentar contra el núcleo de definición simbólicoimaginario de varones rivales. No hay hombres sin mujeres. Esto en un sentido radicalmente concreto; sólo se consigue inscribirse en el mundo de la masculinidad cuando se tiene acceso al bien simbólico, la moneda transaccional por excelencia, que es intercambiada con otros hombres de acuerdo con ciertas reglas aceptadas (por ello pactadas). Los varones feminizados (como aquellos que se inferioriza o invisibiliza por su condición étnica, social o subjetiva), pueden llegar a funcionar también como objetos transaccionales, pero es un 
tema que sólo podemos mencionar y no desarrollar aquí.

Aunque en el nivel más abstracto, personificar significados de feminidad (simbólica) y ser a causa de ello una mujer -o bien un varón simbólicamente feminizado-, implica siempre encarnar tres ideas que mantienen entre sí una tensión irresoluble, pues se trata de ser, a la vez, objeto de deseo, objeto de temor y objeto de desprecio; la manera concreta en que se viste eso es infinitamente variable de una sociedad a otra. Pero, en última instancia, las personas así entendidas son pensadas por el grupo correspondiente como un afuera constitutivo de los seres humanos. Al encarnar lo femenino, las mujeres y los varones feminizados- cumplen para su grupo la función de referentes negativos, de límites. No encarnan a la naturaleza ni a la animalidad, sino a una categoría liminal, que se halla a medio camino entre lo humano y lo no humano. La humanidad es el hombre, desde luego, como lo prueba la generalizada equiparación lingüística de lo masculino con el genérico humano. Las mujeres, al no ser pensadas plenamente como humanas, construyen una identidad tensa y fracturada.

En las fórmulas de actuación de los varones está ritualizándose permanentemente el conjunto de ideas que los instituyen en quienes tienen credenciales suficientes para acceder a las mujeres (según las especifique de manera fina cada grupo) y títulos bastantes para allegarse el reconocimiento de los otros hombres como participantes legítimos del grupo. Cuando una mujer o, peor aún, un grupo de mujeres- actúa en contra de lo que las reglas al uso definen como paradigmático de la feminidad, los varones sienten cuestionada su propia masculinidad. La definición de ellas como femeninas, es lo que les permite a ellos definirse como hombres.

\section{BIBLIOGRAFI'A ${ }^{3}$}

Amorós, Celia (2008). Violencia patriarcal en la era de la globalización: de Sade a las maquilas. En Mujeres e imaginarios de la globalización. Buenos Aires: Homo Sapiens.

\footnotetext{
${ }^{3}$ Se citan en normas APA corregida para visibilizar el trabajo de las autoras.
} 
Amorós, Celia (2009). Espacios de los iguales, espacios de las idénticas. Sobre poder y principio de individuación. En La gran diferencia y sus pequeñas consecuencias...para las luchas de las mujeres (pp. 87-109). Madrid: Cátedra.

Benedict, Ruth (1932). La antropología y el anormal, traducción en línea consultada http://ww2.filo.uba.ar/contenidos/carreras/antropo/catedras/hist teoria antropolo gica/sitio/sitio/pdf/Benedict.pdf

Donnan, Hastings y Fiona Magowan (2010). The Anthropology of Sex. Londres: Bergs Publisher.

Evans-Pritchard, Edward (1937). Witchcraft, Oracles and Magic Among the Azande. Londres: The Clarendon Press.

Evans-Pritchard, Edward (1951). Kinship and Marriage Among the Nuer. Londres: Clarendon Press.

Hutchison, Sharon (1992). The Cattle of Money and the Cattle of Girls among the Nuer. American Ethnologist, Vol. 19, No. 2: 294-316.

Lévi-Strauss, Claude (1969). Las estructuras elementales del parentesco. México: Paidós.

Lévi-Strauss, Claude (1979). Introducción a la obra de Marcel Mauss EN M. Mauss, Sociología y antropología. Madrid: Tecnos, Madrid.

Lévi-Strauss, Claude (1987). La familia EN VV. AA., Polémica sobre el origen y la universalidad de la familia (pp. 9-48). Barcelona: Anagrama.

Martínez Pulido, Carolina (2006). La presencia femenina en el pensamiento biológico. Madrid: Minerva ediciones.

Mauss, Marcel (1979). Sociología y antropología, Madrid: Tecnos.

Mead, Margaret (1982). Sexo y temperamento. Barcelona: Paidós.

Ortner, Sherry (1979). ¿Es la mujer con respecto al hombre lo que la naturaleza con respecto a la cultura?. En Harris y Young (comps.), Antropología y feminismo (pp. 109-132). Barcelona: Anagrama.

Pateman, Carole (1992). The Sexual Contract. Stanford: Stanford University Press.

Rubin, Gayle (1975). The Traffic in Women. Notes on the "Political Economy" of Sex EN Rayana Reiter (comp.) Toward an Anthropology of Women (pp.157-210). Nueva York: Monthly Review Press. 